\title{
Performance Analysis of Filter Bank Multicarrier Cognitive Radio for Physical Layer under Binary Symmetric Radio Fading Channel
}

\author{
A. S Kang \\ Assistant Professor, ECE Deptt \\ Panjab University Regional Centre, \\ Hoshiarpur, Punjab, India
}

\author{
Renu Vig, Ph. D \\ Professor, ECE Deptt \\ Panjab University \\ Chandigarh, India
}

\begin{abstract}
The wireless communication has undergone a revolution due to advancements in technology. For each new user or application to be a part of communication network the preliminary requirement is the allocation of frequency spectrum band. This frequency band is a limited resource and it is impossible to expand its boundaries. So the need is to employ intelligent, adaptive and reconfigurable communication systems which can investigate the requirements of the end user and assign the requisite resources in contrast to the traditional communication systems which allocate a fixed amount of resource to the user under adaptive, autonomic and opportunistic cognitive radio environment. Cognitive Radio(CR) Technology has emerged from software defined radios wherein the key parameters of interest are frequency, power and modulation technique adopted. The role of Cognitive Radio is to alter these parameters under ubiquitous situations. The Spectrum Sensing is an important task to determine the availability of the vacant channels to be utilised by the secondary users without posing any harmful interference to the primary users. In Multicarrier Communication using Digital Signal Processing Techniques, Filter Bank Multi Carrier has an edge over other technologies in terms of Bandwidth and Spectral Efficiency. The present paper deals with the Multi Rate FIR Decimation and Interpolation Filter approach for physical layer of Cognitive Radio under Binary Symmetric fading channel environment.
\end{abstract}

\section{INTRODUCTION}

The users need for higher data rates is increasing at a faster rate in the world to fulfill their evergrowing demands for acessing the information anytime,anywhere under pervasive radio environment.The amount of available frequency spectrum is however, limited.Radio spectrum is a important natural scarce resource needed for radio frequency communication. The radio spectrum is omnipresent in the form of invisible waves and is used by innumerable technologies which ultimately affects the most of the aspects of our daily life. The rf spectrum is a multidimensional concept having features useful for dynamic spectrum access and transmit power control.Radio frequency,Bandwidth,Modulation technique,Power Allocation Scheme and transmit diversity etc influence the performance enhancement of any wireless communication system based on 4G.There are immense areas having vital applications of radio spectrum in defence,public safety and emergency network situations.For the sake of achieving the higher efficiency of a communication system,the following two important physical characteristics play a crucial role in wireless domain.Effective communication of two radio stations at same frequency and the susceptibility of two stations operating within same geographical area to the mutual interference leading to the degradation in the quality of communication taking place between them.Cognitive radio was invented with brain for intelligence of its own with capability of decision making to provide a unique solution to the problem of spectrum underutilization.CR can sense the surrounding environment and depending upon the information as well as requirement of situation needs and alters its physical layer parameters after reconfiguration [1].Fig 1 shows the development of Cognitive Radio technology.

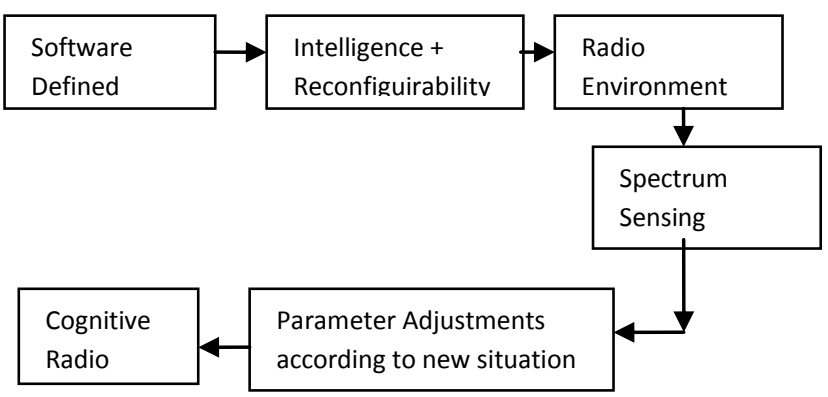

Fig 1.Development of Cognitive Radio

\section{FUNCTIONS OF COGNITIVE RADIO}

The following capabilities are needed for the functionality of cognitive radio. Flexibility and Agility, Sensing, Learning \& Adaptability.The following four functions are mainly performed by cognitive radio.

[1]Spectrum Sensing :It determines the status of the spectrum and detects the presence of primary users.

[2]Spectrum Management:It allocates the available frequency channels to unlicensed secondary users.

[3]Spectrum Sharing:It shares the resources for secondary users.

[4]Spectrum Mobility:Whenever the primary user wants to have the access to channel that secondary user is using,then secondary user immediately vacates that channel for Primary user,thereby switching on to some other vacant channel available nearby [2].Fig.2 shows the Block Diagram of Cognitive Radio Operation. 


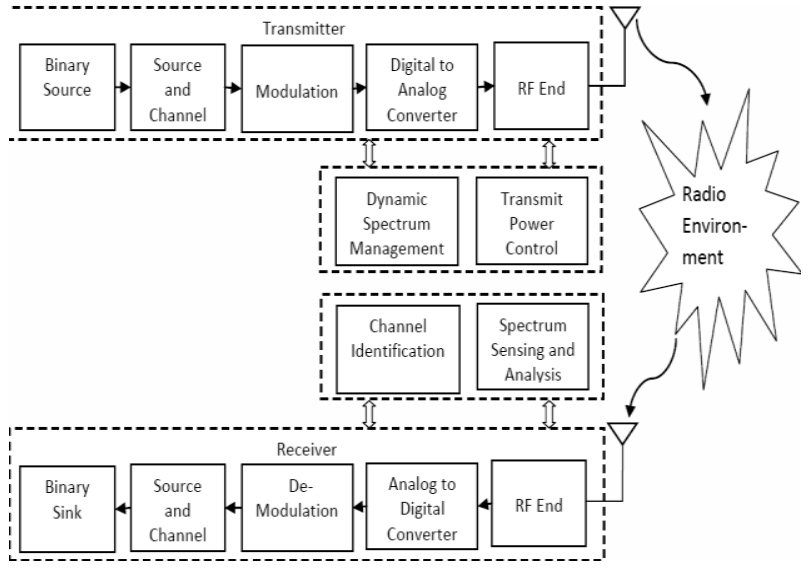

Fig.2 Block Diagram of Cognitive Radio Operation

The OSI model of CR is presented below in figure 3.A binary symmetric channel (or BSC) is a common communications channel model used in coding theory and information theory. In this model, a transmitter wishes to send a bit (a zero or a one), and the receiver receives a bit. It is assumed that the bit is usually transmitted correctly, but that it will be "flipped" with a small probability (the "crossover probability")

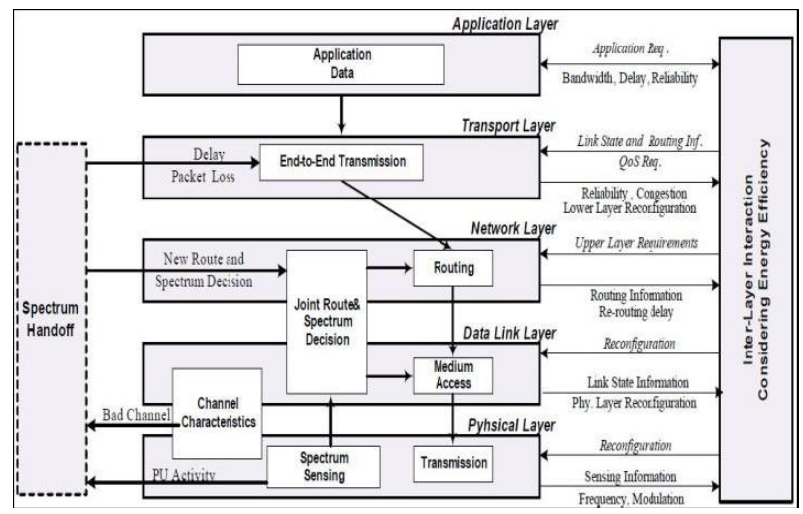

Fig.3 Interaction Between Varoius Layers of OSI Model:Communication and Dynamic Spectrum Management

This channel is used frequently in information theory because it is one of the simplest channels to analyze. The channel model we have used is the binary symmetric channel (BSC) which takes a binary input and with probability $p<1=2$ switches it. This is a good model for deep space communications but not so good for hard drives or for terrestrial communications where errors come in bursts.

\section{ROLE OF DIGITAL FILTER BANKS FOR NEXT GENERATION MULTICARRIER COMMUNICATION SYSTEM}

Multirate DSP is required in digital systems where more than one sampling rate is required.The applicability of multirate signal processing approach is in the areas of Communication Systems,Speech \& Audio Processing systems,Antenna \& Radar Systems. Advanced technique on multirate signal processing can be applied for information processing digitally due to the advantages like Lesser computational requirements,Less storage for filter coefficients, Less finite arithmetic effects,Low requirement of filter order in multirate application,Less sensitivity to filter coefficient length[4-5].

\section{RELATED WORK: SIGNAL PROCESSING PROSPECTIVES IN COGNITIVE RADIO}

M.Laddomada (2011) presented a multirate approach for the recovery of wideband global navigation satellite system signals. Two or more narrow band front ends were used for collecting the different portions of spectrum of wideband navigation signal that was then reconstructed from its sub band components[6].Mohamed Hamid et al (2010) investigated the MAC layer sensing schemes in CR.Simulated results show that to guarantees as low idle channel search delay as possible a proactive sensing is the best scheme to be used [7].B. FarhangBoroujeny and C. H. (George) Yuen(2009), presents a tutorial review relating the classical works on FBMC systems, developed prior of the era of OFDM, to the main filter bank design approaches used today for FBMC systems. The paper also reviews the recent novel developments in the design of FBMC systems that are tuned to cope with fast fading wireless channels[8].N. Moret and A. M. Tonello (2009) ,address the efficient realization of a filtered multitone (FMT) modulation system. The paper analyzes three different realization structures, presenting also numerical comparisons, and compares the best FMT approach with a cyclically prefixed OFDM system in the IEEE 802.11 wireless LAN channel [9].Ziyang Ju et al (2009), developed a method to diagonalize a doubly dispersive channel in the time-frequency domain using a filter bank approach. The related paraunitary filter bank design problem is formulated as a convex optimization problem, and the performance of the resulting window is investigated under different channel conditions [10].Jun Ma et al (2009) discussed the practical issues involved to build a CR network from perspective of signal processing. The author identified spectrum sensing and spectrum sculpting as the two fundamental capabilities for any CR network to adapt to its environment and provide resilience under adverse conditions [11].H. Zhang et al.(2009), studied channel capacity of cognitive radio (CR) networks using CP-OFDM and FBMC waveforms, taking into consideration the effects of resource allocation algorithms, intercell interference due to timing offsets, and Rayleigh fading. Final results show that FBMC can achieve higher channel capacity than OFDM because of the low spectral leakage of its prototype filter [12].P.Amini and B. Farhang-Boroujeny (2009), develop a packet format for FBMC systems together with algorithms for carrier frequency and timing recovery. Also methods for channel estimation as well as carrier and timing tracking loops are proposed [13].T.H. Stitz et al.(2009), presents a detailed analysis of synchronization and channel estimation methods for FBMC based on scattered pilots. The special problems related to using scattered pilot-based schemes in FBMC are highlighted. The channel parameter estimation and compensation are successfully performed totally in the frequency domain, in a sub channel-wise fashion, which is appealing in spectrally agile and cognitive radio scenarios [14].Tero Ihalainen (2006), introduced a new low complexity per-subcarrier channel equalizer for FBMC transceiver for high-rate wideband communication over doubly-dispersive channel and analyzed its performance. it was shown that the coded error-rate performance of FBMC is somewhat better than that of the OFDM reference [15].Linnea Rosenbaun et al (2006), introduced an approach for synthesizing modulated maximally decimated FIR FBs using the FRM technique. Each of the analysis and synthesis FBs was realised with aid of three filters, one cosine modulation block and sine modulation block. The overall FBs achieve nearly PR with a linear phase distortion function [16].Christian Feldbauer et al (2005) described that in 
single rate systems, only one sampling rate is used whereas in multirate systems the sampling rate gets changed at least once. The paper deals with the realization and analysis of multirate systems [17].Mohammad Abo Zahhad (2003), provided an overview of basic concepts, current state and future directions of uniform and non uniform multirate filter banks and their applications. Different design techniques and algorithms that were of interest were investigated from hardware complexity and reconstructed signal's quality point of view [18]

\section{PROBLEM FORMULATION}

Large parts of assigned spectrum is underutilized while the increasing number of wireless multimedia applications lead to spectrum scarcity.Cognitive radio is an option to utilize non used parts of the spectrum that actually are assigned to primary services.The benefits of cognitive radio are clear in the emergency situations.The current emergency services rely much on the public networks. This is not reliable in public networks where the public networks get overloaded.The major limitation of emergency network needs a lot of radio resources.The idea of applying Cognitive Radio to the emergency network is to alleviate this spectrum shortage problem by dynamically accessing free spectrum resources.Cognitive Radio is able to work in different frequency bands and various wireless channels and supports multimedia services such as voice,data and video.A reconfigurable radio architecture is proposed to enable the evolution from the traditional software defined radio to cognitive radio.So cognitive radio network architecture devices detect the Local Oscillator(LO) leakage and communicate the channel usage to cognitive radios. The performance of existing and proposed architectures assuming passive primary receivers can be compared. So detecting primary receivers for cognitive radio applications has been the main motivation that prompts to choose this problem statement. The present study has its focus on Performance Analysis of Filter Bank Multicarrier (FBMC) based Cognitive Radio (CR) in adaptive, opportunistic, autonomic domain under different strategic conditions of Binary Symmetric Channel.By introducing the techniques to improve the spectral efficiency and minimize the spectrum underutilization and interference along with minimum power consumption and Bit Error Rate (BER), the overall performance of FBMC based CR can be improved.

\section{PERFORMANCE ANALYSIS}

The flowchart for initializing the five carrier frequencies for five users at a sampling frequency with a suitable modulation technique for modulating the users data over a respective frequency band.All the modulated signals are added to create a carrier signal and then spectrum periodogram output plot is obtained for estimating the power spectral density.Further,empty slot allocation is done which is clear from the output plot.When a new user arrives,it is assigned a first spectral hole.If all the slots are reserved,then the user is asked to empty a particular slot.After emptying a slot for allocation,certain amount of noise is added to the user slot just for the sake of analyzing the percentage of attenuation required.Ultimately ,the final output plot is observed for this simulation. This process can be repeated a number of times with different empty slot allocations to different users with different amounts of noise proportions to be added for getting a variation in power versus frequency allocation output plots.

\section{SIMULATION STUDY OF FBMC COGNITIVE RADIO}

Simulink based FBMC-CR Model using Binary Symmetric Channel with FIR Decimation \& Interpolation Multi rate
Filters has been developed and implemented as a CR system design. Effect of various parameters given in the blocks of this simulink model has been studied which is further helpful for achieving results as per Simulation testbed.

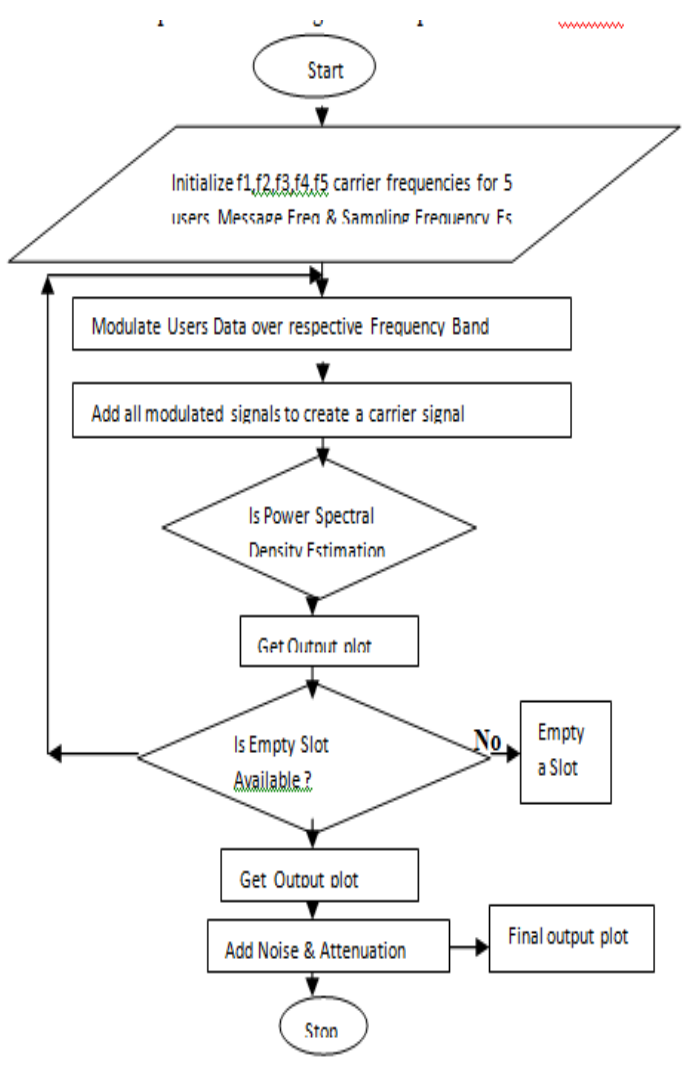

Fig.4 showsSimulationFlowchart.

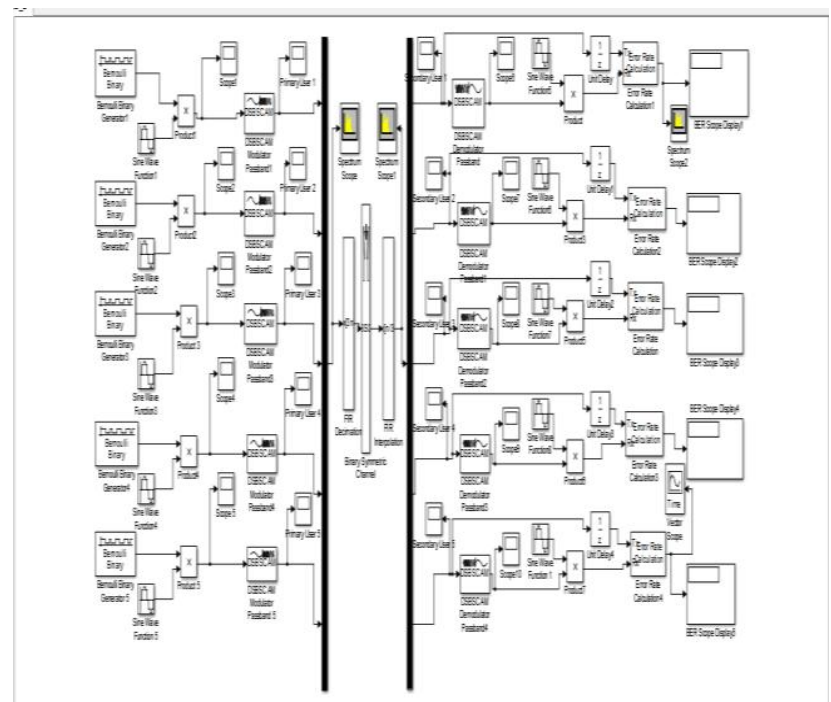

Fig.5 FBMC-CR Simulink Model For Physical Layer Under BSC Prior To Run. 


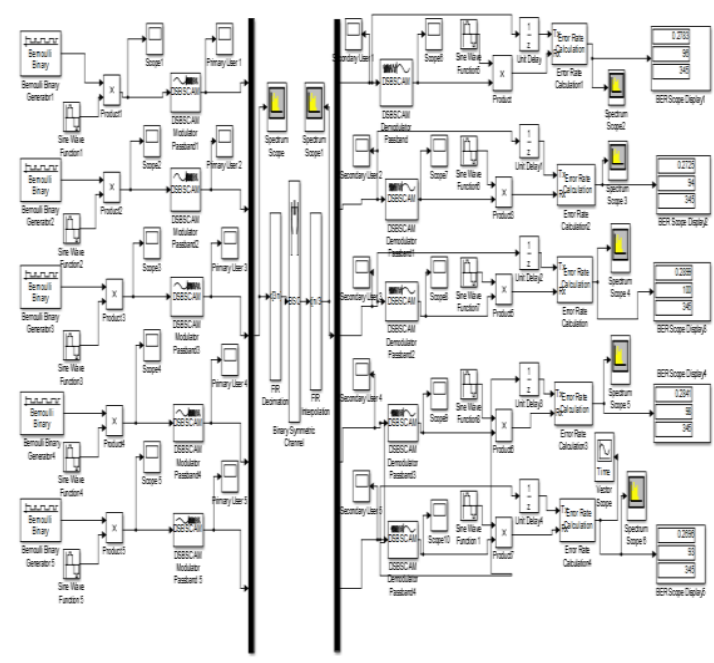

Fig.6 Simulink Model In Running Mode

The various blocks from the Matlab Simulink Library have been used for making the above Simulink Model for FBMC CR using Multirate Filters before and after Binary Symmetric channel.A Code for the proposed study on Cognitive radio has been generated in Matlab R2013a version.

\section{RESULTS AND DISCUSSION}

Simulation model was run over several iterations and output scopes for five primary users as input and five primary users output along with secondary users output scope displays have been obtained which clearly depicts the propagation of sharp spike pulse as input from product modulated output of Bernoulli Binary Generator and Sine wave .Then the primary user input signals are passed through DSBSC AM Modulator Passband whose output is further made to pass through Multiplexer and Demultiplexer.Now in between MUx and DeMux there is a cognitive Radio environment composing AWGN channel with pre and post processing devices in form of FIR Decimation and FIR Interpolation Filters.The output Spectra of these multirate filters used is analysed with the help of Buffered FFT Scopes.Then ,their output passes through all the counter blocks analogous to the Transmitter side of this model.A Unit delay block is introduced for taking into consideration the Tx and Rx error rate calculation block to which a set of five product demodulated outputs is fed as another input.Ultimately,Bit Error rate is computed for showing the effect of attenuation and other technical parameters set in the various simulink parameter blocks on Secondary users.Also the time-amplitude vector scope output is visualized at the end with fifth secondary user in the simulink model.

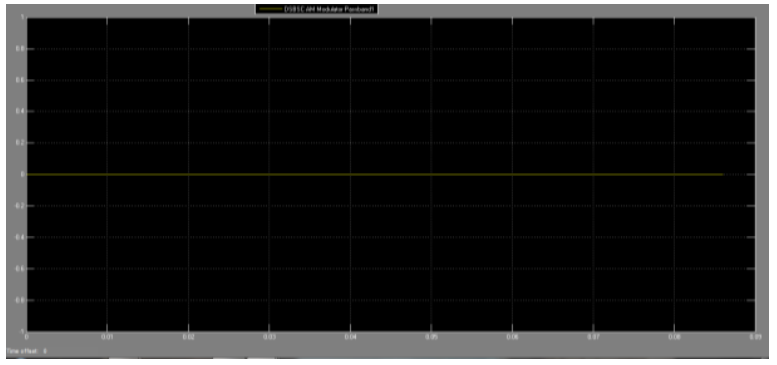

Fig.7 Primary User1 Input Signal

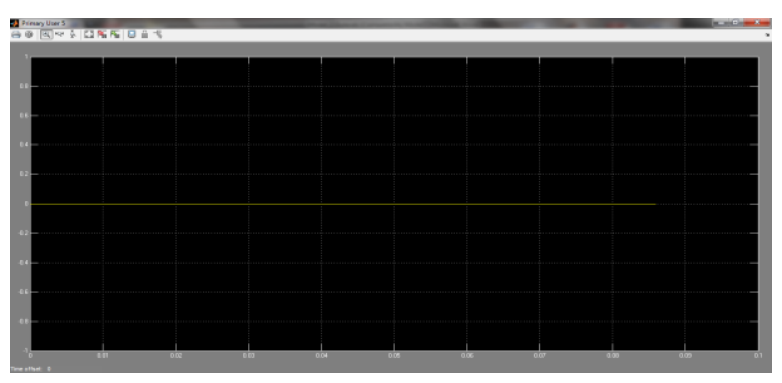

Fig.8 Primary User 5 Input Signal

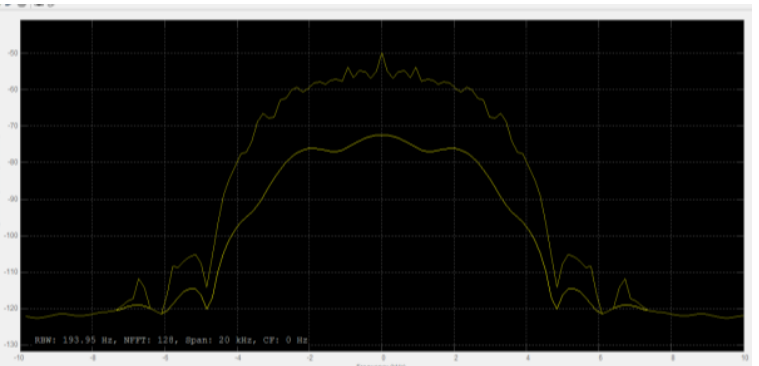

Fig.9 Spectrum Scope 1 Output Display

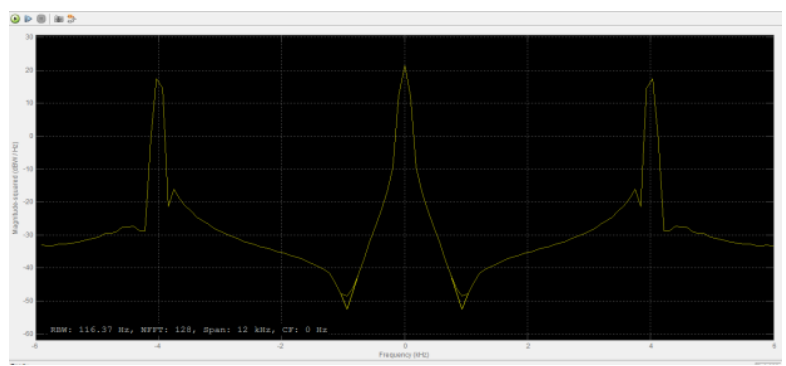

Figure 10. Spectrum Scope2 Output

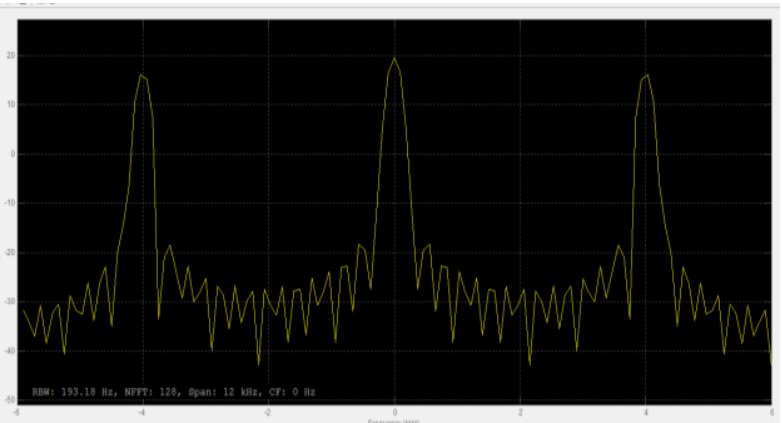

Fig.11 Spectrum Scope3 Output

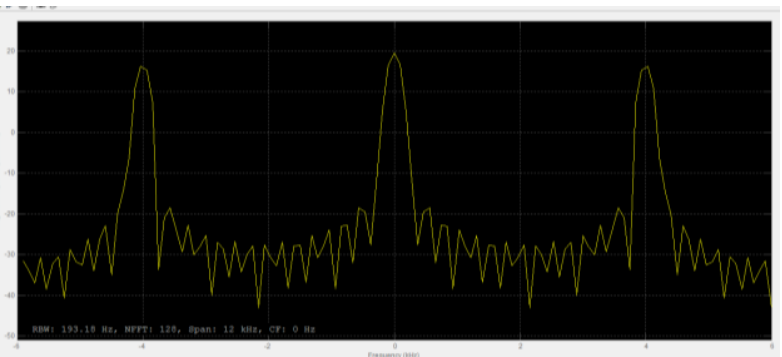

Fig.12 Spectrum Scope6 Output plot 


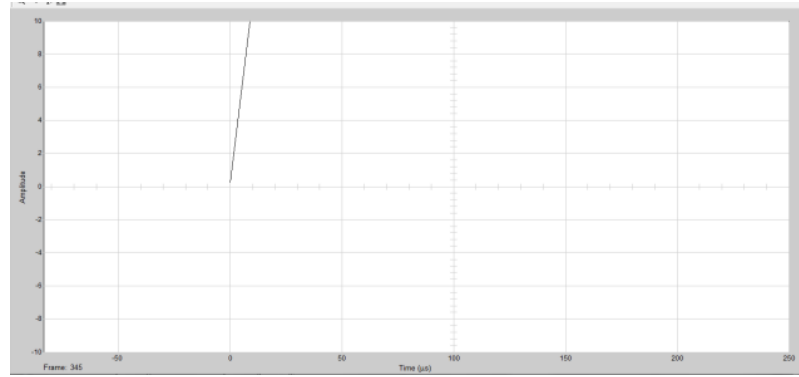

Fig.13 Amplitude-Time Vector Scope Display

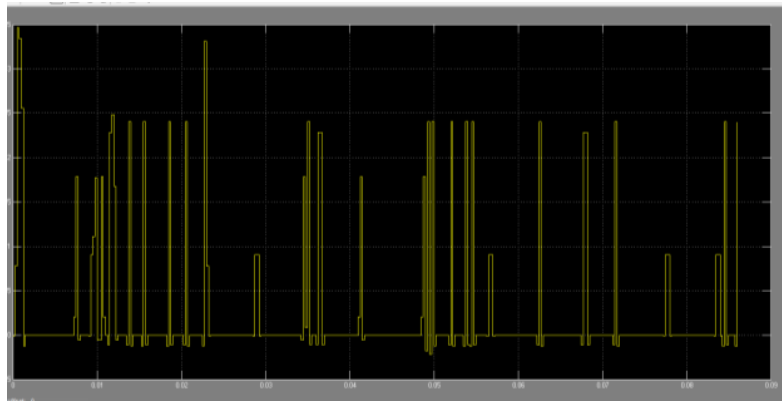

Fig.14 Secondary User1 Output signal display

Buffered-FFT Scope Outputs at three different Instants in a FBMC-CR Simulink model for Physical Layer under AWGN Fading Channel are observed .

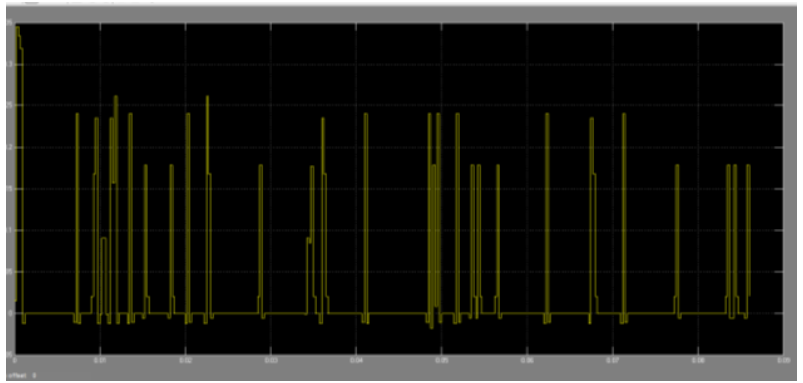

Fig.15 Secondary User5 Output Display

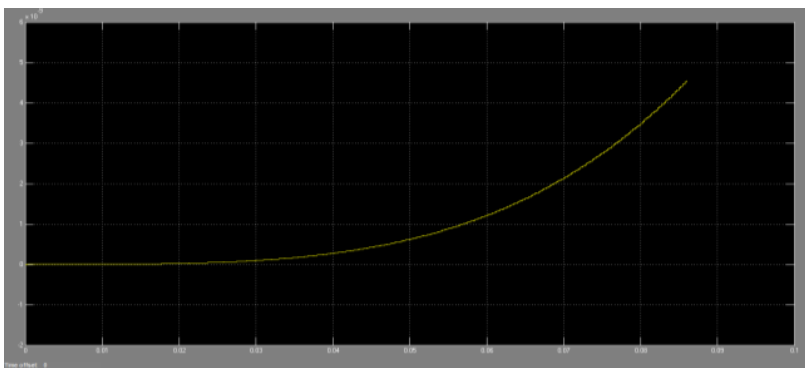

Fig.16 Buffered-FFT Scope 6 Output

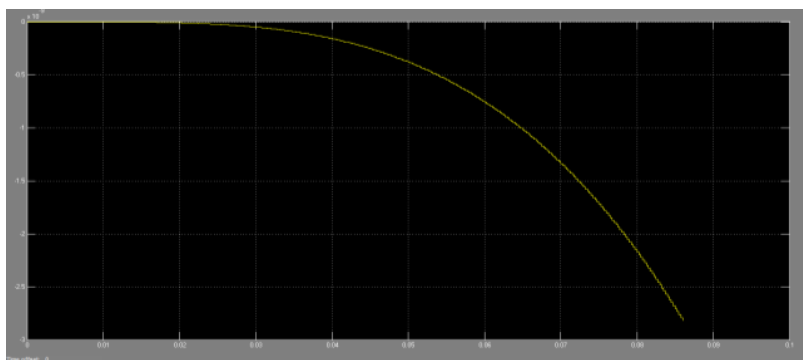

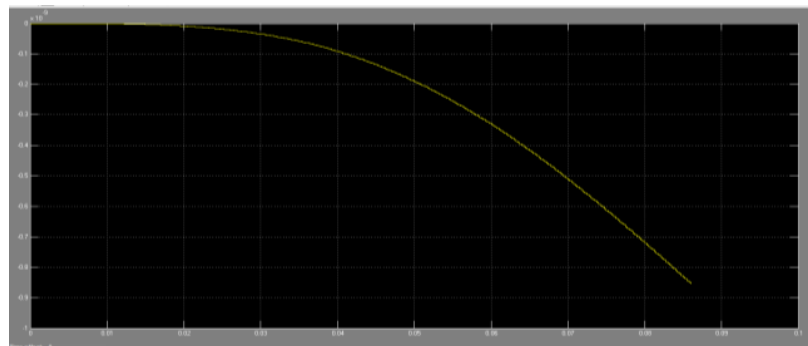

Fig.18 Buffered-FFT Scope8 Output

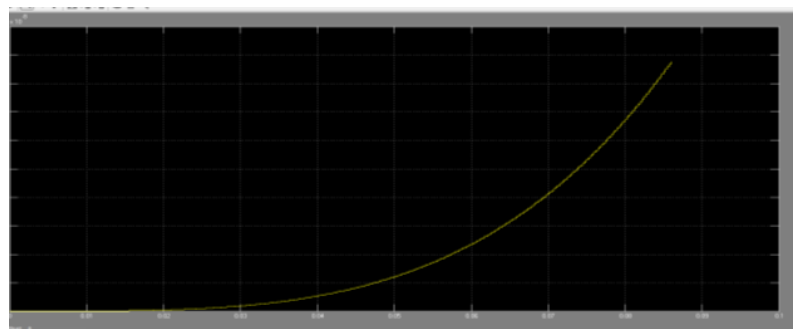

Fig.19 Buffered-FFT Scope10 Output

\section{CONCLUSION}

The present study shows the simulation of cognitive radio system for assessing the dynamic spectrum at the run time.The Magnitude squared $(\mathrm{dBW} / \mathrm{Hz})$ versus Frequency(hz)plots at two instants prior to decimation and after interpolation show the more places of spectrum that are prone to underutilization.Different spectrum scopes show the quick side lobe tail decay occuring with only some peaks,hence showing lesser spectrum underutilization due to less number of white spaces(spectrum holes for cognitive users) which fulfills the need of cognitive radio network for enhanced spectral efficiency.The BER $=0.2788$ has been computed with the aid of simulink model is also a good indicative measure to prove the lesser spectral leakage in the frequency components while passing through the radio frequency channel environment.Hence,an attempt has been made to get better utilization of the bandwidth.

\section{ACKNOWLEDGEMENT}

The first author is thankful to his mentor Dr.Renu Vig and other experts from Panjab University for their valuable discussions and guidance that led to the development of this paper.

\section{REFERENCES:}

[1] J. Mitola and G. Q. Maguire, "Cognitive Radios: Making Software Radios More Personal", IEEE Personal. Communication., Vol. 6, no. 4, pp. 13-18, August 1999.

[2] S.Haykin, "Cognitive Radio: Brain-empowered wireless communications," IEEE Journal on Selected Areas in Communications, Vol. 23, pp. 201-220, Feb. 2005.

[3] I. F. Akyildiz, W.Y.Lee etal., "Next Generation / Dynamic Spectrum Access / Cognitive Radio Wireless Networks: A Survey", Elsevier, Computer Networks 50(2006),Vol.50,no.13,pp. 2127-2159, 2006.

[4] William Krenik etal., "Cognitive Radio Techniques for wide areanetworks", ConferenceProceedings,Texas Instruments,Dallas,USA,pp.409-412,2009.

Fig.17 Buffered-FFT Scope 7 Output 
[5] H.Zhang etal.",Spectral Efficiency Analysis in OFDM and OFDM /OQAM based Cognitive Radio Networks,"IEEE 69th Vehicular Technology Conference, 2009. VTC Spring,pp.1-5,2009.

[6] M. Laddomadaet al., "Advanced Technique on Multirate Signal Processing for Digital Information Processing,"IET Signal Processing,Vol.5, no. 3, pp. 313-315,2011.

[7] Mohammed,Hamid etal.,"On spectrum sharing and Dynamic Spectrum Allocation: MAC layer spectrum sensing in Cognitive Radio Networks"in Procof International Conference on Communication and Mobile Computing,IEEE, pp.183-187,2010

[8] B Farhang Boroujeny etal., "Multicarrier communication techniques for spectrum sensing and communication in cognitive radios,"IEEE Communication Magazine, Vol.46, no.4,pp. 80-85, April 2008.

[9] Nicola Moret and M Tonello, "Design of Orthogonal Filtered Multitone Modulation Systems and Comparison among Efficient Realizations", EURASIP Journal on Advances in Signal Processing, Vol. 2009,pp.1-18,2009.

[10] Ziyang Ju et al., "OptimizedParaunitary Filter Banks for Time-Frequency Channel Diagonalization", EURASIP Journal on Advances in Signal Processing, Vol. 2010, pp.1-12,2010.

[11] JunMa, Li et al.,"Signal Processing in Cognitive Radio", Proc.IEEE,May 2009,Vol.97,No.5,pp.805-823,2009.

[12] H.Zhang et al.,"Spectral Efficiency Comparsion of OFDM/FBMC for uplink Cognitive Radio Networks",
EURASIP Journal on Advances in Signal Processing, Vol. 2010,pp.1-14.

[13] Peiman Aminiand B Farhang Boroujeny, "Packet Format Design and Decision directed Tracking Methods for Filter bank multicarrier Systems", EURASIP Journal on Advances in Signal Processing, Vol. 2010,pp.1-13,2010.

[14] T. H. Stitz et al., "Pilot- Based synchronization and Equalization in Filter Bank Multicarrier Communication “, EURASIPJournal on Advances in Signal Processing, Vol. 2010,pp.1-18,2010.

[15] Terolhalainen, T.H.Stitzet al.,"Channel Equalization in Filter Bank based Multicarrirer Modulation For Wireless Communacations",Hindawi Publishing Corporation,EURASIP Journal on Advances in Signal Processing, Vol. 2007,pp.1-18,2007.

[16] Linnea Rosenbaum et al., "An Approach for synthesis of Modulated M-Channel FIR Filter banks Utilizing the Frequency-Response Masking Technique,"EURASIP Journal on Advances in Signal Processing, Vol. 2007,pp.1-13,2007.

[17] Christian Feldbauer,Marian Kepesi et al., "Multirate Signal Processing",Graz University of Technology, Vol. 1.3.3,pp.1-10,2005.

[18] Mohammed Abo-Zahhad,"Current State and Future Direction of Multirate Filter Banks and their Applications"Digital Signal Processing13(2003),Elsevier science (USA),Vol.13,no.3, pp.495-518, 2003. 